Document downloaded from:

http://hdl.handle.net/10251/165609

This paper must be cited as:

Miragall, M.; Borrego, A.; Cebolla, A.; Etchemendy, E.; Navarro-Siurana, J.; Llorens Rodríguez, R.; Blackwell, SE.... (2020). Effect of an upright (vs. stooped) posture on interpretation bias, imagery, and emotions. Journal of Behavior Therapy and Experimental Psychiatry. 68:1-9. https://doi.org/10.1016/j.jbtep.2020.101560

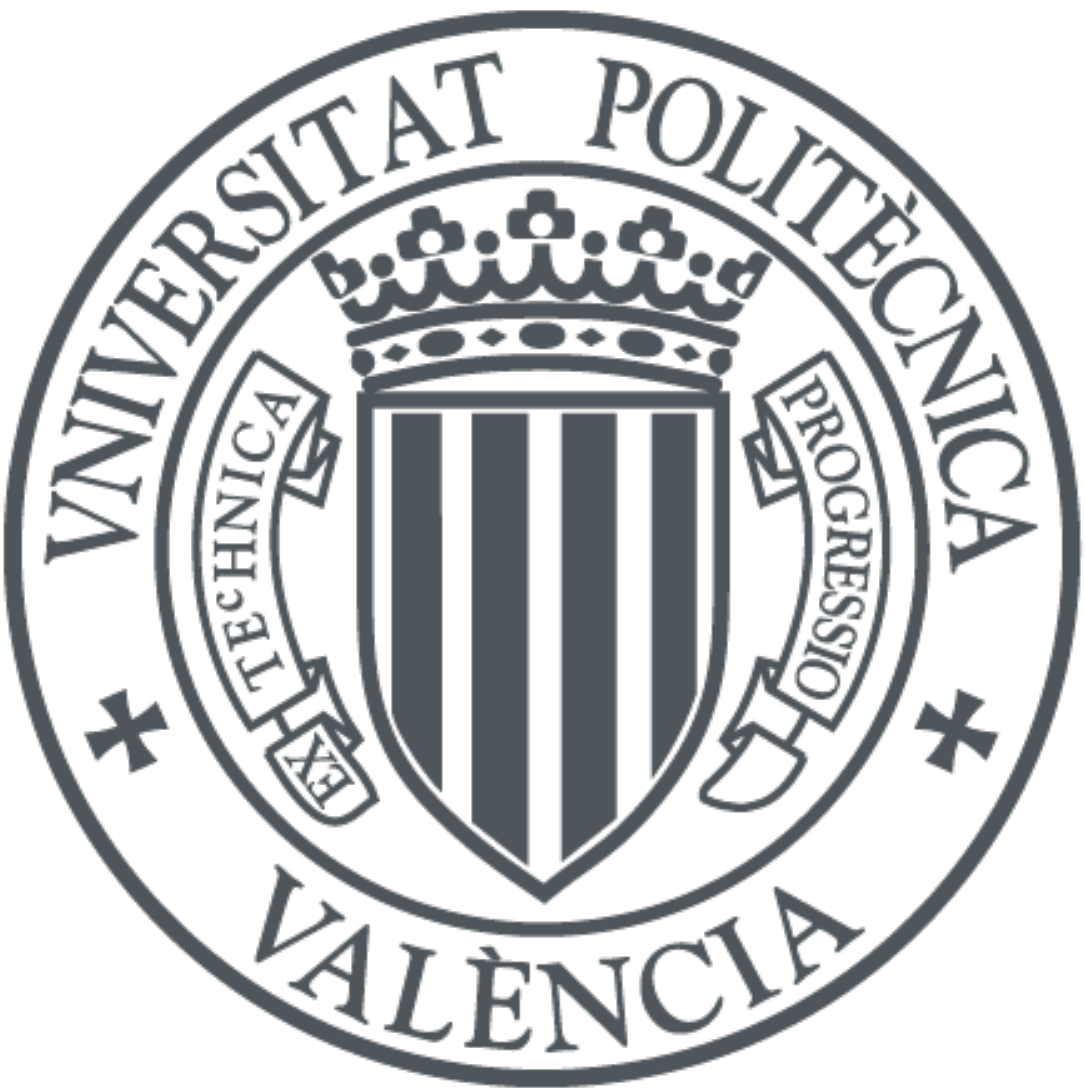

The final publication is available at

https://doi.org/10.1016/j.jbtep.2020.101560

Copyright Elsevier

Additional Information 


\section{Highlights}

- Upright (vs. stooped) posture reduced the negative interpretation bias.

- Upright (vs. stooped) posture increased positive emotions (e.g., optimism).

- Time in upright posture was related to changes in interpretation bias and imagery.

- Depressive symptoms moderated the effect of posture on interpretation bias change.

- Posture interacted with mechanisms involved in the maintenance of depression. 


\begin{abstract}
Background and Objectives: Adopting an upright (vs. stooped) posture has been related to positive effects on emotional and cognitive processes. However, there is no evidence concerning the effect of posture on two key processes associated with the maintenance of depression: interpretation bias and vividness of mental imagery. The objectives were to investigate the effect of adopting an upright (vs. stooped) posture on interpretation bias and vividness of positive and negative mental imagery, and to explore the interplay between these processes and depression-related emotions.
\end{abstract} Methods: The sample consisted of 54 participants $\left(M_{\text {age }}=22.00,64.8 \%\right.$ women), who were randomly assigned to the upright or stooped condition. Participants answered selfreport measures while they were adopting a specific posture. Posture was monitored through inertial technology. Results: Main results were that: upright (vs. stooped) posture led to more positive interpretations of ambiguous information and increased positive emotions related to depression (happiness, optimism and vigor); time in an upright position was associated with change in interpretation bias and vividness of positive mental imagery; and level of depressive symptomatology moderated the effect of posture on the change in interpretation bias. Limitations: Limitations are related to the use of non-clinical sample, the use of short-term measurements, and the lack of an experimental condition adopting the usual posture. Conclusions: Posture interacts with mechanisms involved in the maintenance of depression, as well as with depressionrelated emotions. This study has clinical implications that should be continued explored in order to clarify the role of manipulating the posture in individuals with depressive symptomatology.

Keywords: interpretation bias; mental imagery; upright posture; stooped posture; depression. 


\title{
Effect of an upright (vs. stooped) posture on interpretation bias, imagery, and emotions
}

\author{
Marta Miragall ${ }^{\mathrm{a}, \mathrm{b}^{*}}$, Adrián Borrego ${ }^{\mathrm{c}}$, Ausiàs Cebolla ${ }^{\mathrm{a}, \mathrm{b}}$, Ernestina \\ Etchemendy ${ }^{d}$, Jéssica Navarro-Siurana ${ }^{\mathrm{a}}$, Roberto Llorens ${ }^{\mathrm{c}, \mathrm{e}}$, Simon \\ Blackwellf , and Rosa $\mathrm{M}^{\mathrm{a}}$ Baños ${ }^{\mathrm{a}, \mathrm{b}}$
}

${ }^{a}$ Department of Personality, Evaluation and Psychological Treatment. University of Valencia, Valencia, Spain.

${ }^{b}$ CIBER Fisiopatología Obesidad y Nutrición (CIBEROBN). Instituto Carlos III, Spain.

${ }^{c}$ Neurorehabilitation and Brain Research Group, Instituto de Investigación e Innovación en Bioingeniería. Universitat Politècnica de València, Valencia, Spain.

${ }^{d}$ Department of Psychology and Sociology. University of Zaragoza, Teruel, Spain.

e Servicio de Neurorrehabilitación y Daño Cerebral de los Hospitales Vithas-NISA. Fundación Hospitales NISA, Valencia, Spain.

${ }^{f}$ Department of Psychology, Ruhr-Universität Bochum, Germany.

All author's e-mail addresses (in order): marta.miragall@uv.es; adborgon@i3b.upv.es; ausias.cebolla@uv.es; ernestinae@unizar.es; jesnasiu@alumni.uv.es; rllorens@i3b.upv.es;

\section{Simon.Blackwell@ruhr-uni-bochum.de; Rosa.Banos@uv.es}

*Corresponding author: Department of Personality, Evaluation, and Psychological Treatment. University of Valencia, Spain. Avda. Blasco Ibañez, 21. 46010, Valencia, Spain. E-mail address: marta.miragall@uv.es. Telephone number: +34963864412 


\section{Acknowledgments}

This work was supported by the Ministerio de Economia y Competitividad of Spain under grant "BODYTA" (PSI2014-51928-R), and BES-2014-068218; and by Universitat Politècnica de València (Grant PAID-10-16). The funding source had no involvement in the study design, in the collection, analysis and interpretation of data, in the writing of the report, or in the decision to submit the article for publication. CIBERobn is an initiate of the ISCIII. 


\section{Effect of an upright (vs. stooped) posture on interpretation bias, imagery, and emotions}

\section{Introduction}

Major depression is a mental disorder comprised of emotional, cognitive, and behavioral symptoms, including depressed mood or a loss of interest or pleasure, and a set of further specific symptoms (e.g., change in activity and/or sleep, concentration, fatigue) (American Psychiatric Association, 2013). The World Health Organization (WHO) $)^{1}$ has estimated that more than 300 million individuals of all ages -equivalent to $4.4 \%$ of the world's population- were depressed in 2015, constituting the largest contributor to global disability and to deaths by suicide (WHO, 2017).

Evidence has suggested an important role for cognitive biases, such as in attention (e.g., attending to emotionally congruent cues), memory (e.g., recalling more unpleasant memories), and interpretation (e.g., interpreting ambiguous events in a more negative manner), in the context of depression (Evaraert et al., 2012; Gotlib and Joorman, 2010; Mathews and Macleod, 2005). In fact, several cognitive models have implicated these cognitive biases as mechanisms involved in the etiology and maintenance of depression (e.g., Clark et al., 1999; Ingram, 1984; Joorman et al., 2007; LeMoult and Gotlib, 2019; Platt et al., 2016; Williams et al., 1988, 1997). More specifically, the relationship between interpretation biases and depression has been supported by a recent meta-analysis conducted by Everaert et al. (2017), which found a

\footnotetext{
${ }^{1}$ Abbreviations: $\mathrm{WHO}=$ World Health Organization; ICS = Interacting Cognitive Subsystems; BDI-II = Beck Depression Inventory II; AST-D-II = Ambiguous Scenarios Test for depressionrelated interpretation bias; PIT $=$ Prospective Imagery Test; ES = Emotions' Scale; POMS = Profile of Mood States; CBM = Cognitive Bias Modification.
} 
medium overall effect size for this relationship in different samples (e.g., individuals with clinical depression or reporting elevated depressive symptoms).

In addition to the role of the interpretation biases in depression, it has also been suggested that individuals with depression experience an excess of intrusive involuntary negative mental imagery, an impoverishment in the quality (e.g., vividness) of deliberately generated future-oriented positive imagery, and difficulty in voluntarily generating specific images of the past or future (Holmes et al., 2016). Indeed, the psychopathological model proposed by Holmes et al. (2009) has pointed out that both disturbances in mental imagery (negative intrusive imagery and lack of positive imagery) and negative interpretation bias may contribute to maintain depression independently (e.g., through negative interpretations of the events, or the preponderance of negative intrusive imagery of past or future events). Moreover, this model proposes a combined cognitive biases hypothesis for explaining how disturbances in mental imagery and negative interpretation bias contribute to maintain and exacerbate depression. According to this model, when these processes interact (e.g., a negative interpretation takes the form of a negative image, instead of a verbal thought, or a negative intrusive image is interpreted in a dysfunctional manner), this would contribute to both the maintenance of depressed mood and its exacerbation.

While the above accounts concern the influence of cognitive processes on emotion, embodied cognition and emotion theories have highlighted the role of sensory or motor bodily states in shaping cognitive and emotional processes (Barsalou, 2008; Niedenthal, 2007; Winkielman et al., 2015). One of the psychological mechanisms for explaining the embodied cognition effect has been proposed by Körner et al. (2015) and is called direct state induction. According to this mechanism, bodily states can directly modify the state of mind, the feelings, or the information processing of an individual, 
without the mediation of other cognitive mechanisms. Indeed, a growing body of literature supports the relationship between the manipulation of the body posture and emotional and cognitive processes. A recent study with $p$-curve analyses, based on a systematic review of 55 studies related to the uprightness and expansiveness of the posture, has shown robust evidence for the effect of posture on feelings of power (currently the most studied variable related to the effect of posture), emotions, affect, mood recovery, retrieval and recall of positive (vs. negative) memories, and selfevaluations (Cuddy et al., 2018).

In this regard, several experiments support the effect of the adoption of upright (vs. stooped) postures in cognitive processes, such as in the retrieval and recall of specific memories. In an experiment conducted by Michalak, Mischnat, and Teismann (2014) participants with major depression were asked to create a visual scene for positive and negative words by imagining themselves in connection with the presented word while they were maintaining an upright or stooped posture. After that, participants seated in a stooped posture recalled more negative words, while participants seated in an upright posture showed a balanced recall of positive and negative words. Other studies with non-clinical samples have shown similar results. For instance, Wilson and Peper (2004) assessed whether it was easier to generate positive and negative past events in an upright or stooped body posture, finding that positive past events were easier to recall in the upright posture; or Tsai et al. (2016) reported that evoking positive events required more effort or arousal (e.g., increase in high-frequency oscillatory activities in electroencephalogram patterns) while in a stooped (vs. an upright) posture.

Moreover, it is worth mentioning specific studies that show that the adopted posture seems to have different effects on the emotional symptoms that may arise in depression or on mood recovery. Wilkes et al. (2017) found that upright (vs. the usual) 
posture may increase positive affect, reduce fatigue, and decrease self-focus in a speech task in people with mild to moderate depression. In the same line, Veenstra et al. (2016) found that posture can play an important role in recovery from a negative mood. Participants who adopted a stooped (vs. upright) posture recovered less from their negative mood after a negative mood induction, regardless of whether participants performed cognitive reappraisal or not.

The role of the body in the maintenance of depression has been highlighted in the Interacting Cognitive Subsystems (ICS) approach (Barnard and Teasdale, 1991). This is a theoretical comprehensive model of the organization and function of the resources underlying individuals' cognition, which proposes that there are several cognitive subsystems specialized in handling different information. One of them is the "implicational subsystem", which is directly linked to emotion, and encodes schematic models that processes more implicit, generic, and holistic levels of meanings (e.g., global negative view of self). It integrates information from two other subsystems: the "propositional subsystem” (which encodes specific, explicit, factual, or conceptual meanings) and the "body state subsystem" (which encodes sensory inputs from taste, smell, touch, pain, and proprioception). According to this approach, depressed states arise when depressed-related schematic models are produced from negative propositional meanings and negative body states, and are maintained because there is an interlocked negative feedback loop across the different subsystems.

Despite the empirical evidence and theoretical approaches that highlight the effects of some bodily states on emotional and cognitive processes, so far no studies have analyzed the effect of posture on two key processes associated with the maintenance of depressed mood according to Holmes et al. (2009): interpretation bias and abnormalities in imagery. Hence, the main objective of this study was to analyze 
the effect of posture on the processing of ambiguous information and the ability to generate vivid mental imagery, as well as on several self-reported positive and negative emotions, vigor, fatigue, and depressive state during the adoption of an upright (vs. stooped) posture. To do so, a non-clinical sample of individuals across a range of levels of depression severity, from absent to moderate symptoms of depression, was used. Moreover, the thoracic spine flexion was recorded using inertial technology to ensure that participants in each condition held the appropriate posture.

According to the effects of posture on memory bias and emotions found in previous studies (e.g., Michalak et al., 2014; Wilkes et al., 2017), it was expected that the upright (vs. stooped) posture would enhance the accessibility of "positive" cognitions and emotions. Hence, the main hypothesis of this study was that adopting an upright (vs. stooped) posture would lead to individuals interpreting the ambiguous scenarios more positively, imagining the positive future scenarios more vividly, imagining the negative future scenarios less vividly, as well as experiencing more positive emotions (e.g., happiness), less negative emotions (e.g., sadness), more vigor and less fatigue and depressive state.

Consistent with the focus of the present study of analyzing the role of the body in the maintenance of depressed mood, the secondary aims of this study were to explore the interactions between posture, interpretation bias, imagery, and depression-related emotions. Thus, one secondary objective was to explore the relationship between the percentage of time spent in an upright posture (measured by inertial technology) and the change in interpretation bias, imagery, and emotions. Another secondary objective was to analyze for whom (considering the depressive symptomatology as moderator variable) the upright or stooped posture may have a greater effect on changing interpretation bias and mental imagery. Nevertheless, given the novelty of this study 
and the exploratory nature of these secondary objectives, no specific hypotheses were generated.

\section{Method}

\subsection{Participants}

A total of 26 participants per condition were planned to be included in the sample to detect a medium-to-large effect size, and taking into account an alpha error of .05 , and statistical power of .80 . This effect size was chosen with reference to related studies with similar outcomes (e.g., Michalak et al., 2014; Nair et al., 2015; Wilkes et al., 2017). Four more participants were recruited to preserve statistical power in case of exclusions. The sample size was determined using $G^{*}$ Power (Faul et al., 2007).

A sample with a wide range of level of depressive symptoms was used, as we were interested in exploring the effects posture on interpretation bias, imagery, and emotion in a non-clinical sample that included participants with dysphoria (people who are not suffering currently depressive symptoms, but are at risk of developing them). The eligibility criteria for the present study included: 1) to have a score between 0 and 27 in the Beck Depression Inventory II (BDI-II); and 2) to be a native Spanish speaker. The exclusion criteria were: 1) to be currently under psychological treatment; and 2) risk of suicidality ( $>1$ on the suicidality item of the BDI-II).We placed an upper limit on the severity of depressive symptoms (i.e. $<28$ on the BDI-II and no current suicidality) to reduce the risk of including individuals with highly significant levels of clinical or functional impairment, where there may be a qualitative rather than only quantitative difference in the relationships between the variables of interest.

The sample was recruited in the Psychology degree classrooms at the University of XXXXXXX. The screening questionnaires (sociodemographic information and BDI- 
II) were completed by 259 students, and those who met the inclusion and exclusion criteria (158) were invited to participate by phone. However, 59 participants did not respond to the phone call, 36 declined to participate in the experiment, and 7 did not come to the appointment.

The total sample was composed of 54 students with no/minimal, mild, or moderate depressive symptomatology (total score in BDI-II $\leq 28$ ). The mean age of the sample was $22.00(S D=2.84$, range: 18 to 32$), 64.8 \%$ were women, and the mean score on the BDI-II was $9.39(S D=7.60$, range: 0 to 26$)$. Regarding exclusions, 2 participants were excluded due to knowing the specific objective of the study (e.g., the link between posture, interpretation bias, and imagination) or scoring $>28$ on the BDI-II on the day of the experiment.

All participants signed the informed consent documents before filling the screening questionnaires and starting the experiment, in accordance with the Declaration of Helsinki. The Ethics Committee at the University of XXXXXXX approved the study.

\subsection{Measures and materials}

Sociodemographic information. An ad-hoc questionnaire was made to collect information regarding age, sex, work status, education, and previous depressive episodes.

Depressive symptomatology: The Beck Depression Inventory-II (BDI-II). The BDI-II (Beck et al., 1996; Sanz et al., 2003) is a self-report questionnaire consisting of 21 items that measure the presence of depressive symptoms in the last 2 weeks. In this sample, the internal consistency was adequate $(\alpha=.89)$. 
Interpretation bias: The Ambiguous Scenarios Test for depression-related interpretation bias (AST-D-II). The AST-D-II (Rohrbacher and Reinecke, 2014) consists of two 15-item parallel versions (i.e. two versions, form $\mathrm{A}$ and form $\mathrm{B}$, that are equivalent both in terms of content and psychometric properties), in which the items are ambiguous scenarios (e.g., future situations, past experiences) that allow either a positive or a negative interpretation (e.g., example of scenario in form A: "You are going to see a very good friend at the station. You have not seen them for years. You feel emotional, thinking about how much they might have changed"; example of scenario in form B: "You are hosting a dinner party for 10 people and got pretty stressed out while preparing the food. You can tell from the initial reaction of the guests how they like the food.). Form A and form B of the AST-D-II measure the Beck's cognitive triad -the tendency to make negative interpretations of ambiguous "future situations", "past experiences", or "situations concerning one's own skills and performance-, but with different scenarios. Participants should imagine each situation as vividly as possible and rate the level of pleasantness on an 11-point Likert scale $(-5=$ extremely unpleasant; $+5=$ extremely pleasant). A Spanish adaptation carried out by the authors was used, showing an adequate internal consistency across administrations ( $\alpha$ ranging from .74 to .81$)$.

Mental imagery vividness: Prospective Imagery Task(PIT). The PIT (Holmes et al., 2008; Stöber, 2000) measures the vividness with which individuals can imagine 10 positive and 10 negative events in their future (e.g., "You will have a serious disagreement with your friend", "You will do well on your course”). Participants should imagine each scenario happening to them and rate the vividness of their image on a 5-point Likert scale $(1=$ no image at all; $5=$ very vivid $)$. Two composite indexes were calculated for the negative future scenarios (negative-PIT), and the positive future 
scenarios (positive-PIT). A Spanish adaptation performed by the authors was used, that showed adequate internal consistency across administrations ( $\alpha$ ranging from .74 to .78 for negative-PIT, and from .78 to .83 for positive-PIT).

Happy, Sad, Optimistic, Hopeless, Anxious, Relaxed: Emotions'Scale (ES). The ES consisted of three positive emotions (happy, optimistic, relaxed) and three negative emotions (hopeless, anxious, sad) that have been used in a previous published study protocol (Blackwell et al., 2018), in which individuals are asked rate to what extent they felt each emotion "right now/in the last few minutes" on a 5-point Likert scale $(1=$ not at all; 5 = extremely) taken from the "state" version of the Positive and Negative Affect Schedules (Watson et al., 1988). Two composite indexes were calculated for positive and negative emotions in order to reduce type error I. Since the scale had not been previously validated, the inter-item correlations between the different emotions in the pre-scores were calculated in order to investigate whether the items "happy, optimistic, relaxed" were measuring the same dimension of positive emotions, and the items "hopeless, anxious, sad" were measuring the same dimension of negative emotions. Inter-item correlations were: anxious and hopeless $(r=.51)$, anxious and $\operatorname{sad}(r=.26)$, hopeless and sad $(r=.59)$, optimistic and happy $(r=.73)$, relaxed and optimistic $(r=$ $.05)$, and relaxed and happy $(r=.22)$. Since the inter-item correlation between emotions were adequate, except for "relaxed and optimistic" -which was poor $(r<.20)$ (Piedmont, 2014) - two composite indexes were calculated for positive (excluding "relaxed") and negative emotions. Hence, relaxation was computed separately, as this item was only poorly correlated with "optimistic", and consequently, it might not be representative of the same content domain. The internal consistency was adequate for negative ES ( $\alpha$ ranging from .59 to .70 ) and for positive ES ( $\alpha$ ranging from .84 to .89 ) across the administrations. 
Fatigue, Vigor, and Depressive state: The Profile of Mood States (POMS). The POMS (McNair et al., 1971; Andrade et al., 2013) is a self-reported questionnaire, in which individuals were asked to rate to what extent they felt each emotion "right now/in the last few minutes" on a 5 -point Likert scale $(0=$ not at all; $4=$ extremely $)$. Only the subscales related to our hypothesis were used: fatigue, vigor, and depressive state. Internal consistency was adequate for fatigue ( $\alpha$ ranging from .88 to .90 ), vigor ( $\alpha$ ranging from .92 to .94 ), and depressive state ( $\alpha$ ranging from .84 to .85 ) across the administrations.

Thoracic spine flexion: Smartphone with a gyroscope. This inertial technology was used to assess the curvature of the thoracic spine, in which $0^{\circ}$ was equivalent to sitting totally upright. An ad-hoc mobile application was developed to collect inertial data time series during the experiment. The smartphone was fixed on the participants' back at the thoracic spine level (T3-T4), between the scapulae, with an elastic band that surrounded the shoulders.

\subsection{Procedure}

To cover the main objective of the study, the researcher explained that the aim of the study was to validate a motion sensor that measures the flexion of the thoracic spine while people are doing different tasks. Participants filled out a sociodemographic questionnaire, the BDI-II ${ }^{2}$, the AST-D-II, the PIT, the ES, and the POMS through an online survey tool before adopting the posture (pre-assessment measures).

Participants were then randomly assigned to one of the two experimental conditions: upright posture $(n=28)$ or stooped posture $(n=28)$. The manipulation of body posture was performed according to Michalak et al. (2014). The instructions for

\footnotetext{
${ }^{2}$ Participants filled in the BDI-II in the day of the experiment in order to assure that they met the first inclusion criterion (scoring $\leq 28$ in the BDI-II).
} 
participants in the upright condition were: "You should sit in a chair without tension in your legs or your feet, elevating your chin, with your back and neck straight, and bringing your shoulders back”. In contrast, the instructions for participants in the stooped condition were: "You should sit in the chair without tension in your legs or your feet, dropping your head, letting your rib cage and shoulders fall, and stooping your back”. (Figure 1). To ensure the correct body posture in each condition, the laptop was placed at approximately 1 meter from the chair, at the eye level in the upright condition, and on the floor in the stooped condition. Participants were also equipped with the smartphone and were required to hold a completely upright posture to calibrate the application. The inertial data registered in this posture was used as reference value for the inertial data recorded during the experiment. Participants adopted the upright or stooped posture for 2 minutes before answering the post-assessment measures, as in Carney et al. (2010) or Cuddy et al. (2015). Next, they answered the AST-D-II, the PIT, the POMS, and the ES (post-assessment measures) while continuing to hold the posture. A wireless mouse was given to the participants in order to answer the questionnaires, and participants in both experimental conditions rested the mouse on their own leg to move it. The AST-D-II and the PIT were recorded with a female voice and were presented aurally ${ }^{3}$ at both time points. Participants answered the AST-D-II and the PIT in a counterbalanced order.

In summary, first, participants completed the pre-assessment measures (without manipulating the posture); second, the posture of the posture was manipulated for 2 minutes; and finally, participants completed the post-assessment measures (while they were maintaining the posture). Thus, participants held the posture for approximately 15-

\footnotetext{
${ }^{3}$ It was decided to use this kind of presentation for practical reasons (in order to not interfere with the body posture). However, the AST-D-II and the PIT are normally answered by reading the items, not hearing them.
} 
20 minutes in total (considering the two minutes before answering the questionnaires and the time answering the post-assessment questionnaires). At the end of the study, participants were asked to explain the hypothesis they had regarding the objective of the study. 

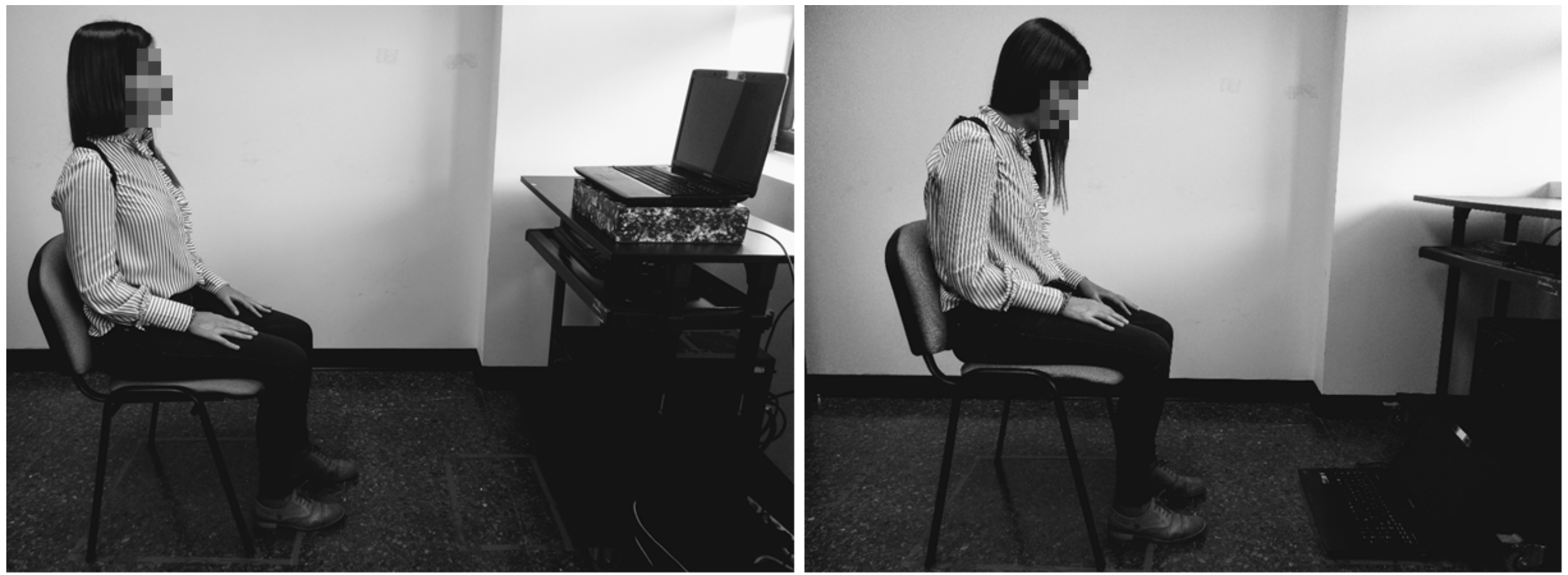

Fig. 1. Upright and stooped posture adopted during the experiment

Note. This body manipulation was based on Michalak et al. (2014). 


\subsection{Data analyses}

All statistical analyses were performed using the SPSS v.24. First, descriptive statistics, independent-samples t-tests, and chi-square tests were performed to test whether there were significant differences between conditions at baseline in sociodemographic variables, levels of depressive symptomatology, and variables investigated in this study.

Second, an independent-sample t-test was performed to test whether there were significant differences in the percentage of time that participants adopted an upright posture in each condition. To do so, since there is no cut-off in the literature that indicates which degree of inclination of the thoracic part of the back (measured by inertial technology fixed at the thoracic spine level) corresponds to an upright/stooped posture, the following procedure was carried out: (1) the range of the degree of inclination of the thoracic part of the back for all the sample was calculated (which ranged from $-2.81^{\circ}$ to $37.96^{\circ}$ ); (2) the median of the degrees of this range was calculated in order to establish a cut-off that separates the higher half of the sample from the lower half (which was $7.34^{\circ}$ ); (3) considering the median as a cut-off, two ranges representing the upright posture and the stooped posture of this sample were generated (which were $-2.81^{\circ}-7.33^{\circ}$ for the upright posture, and $7.34^{\circ}-37.96^{\circ}$ for the non-upright posture); and finally (4) the percentage of time that the participant was in an angle equivalent to an upright posture was calculated ([Time of monitoring maintaining an inclination of the back within the range $-2.81^{\circ}-7.33^{\circ}$ T Total time of monitoring] $\mathrm{x}$ 100). The percentage of time in the upright posture was calculated in order to avoid distorted results because of possible motion-related artifacts while maintaining the allocated posture. That is, as the participants were not immobilized during the adoption of the body posture in order to reduce the artificiality of the situation, they could occasionally move during the experiment (e.g., participants in the stooped condition 
occasionally straighten up or participants in the upright posture occasionally slumped), that might cause extreme (and not representative) values of the curvature of the thoracic spine adopted during the experiment. For this reason, the percentage of time in the upright posture -instead of other statistical indices, such as the mean of the curvature of the thoracic spine- may be more accurate as a measure of the adopted posture.

Third, to check the effects of the posture on interpretation bias, mental imagery vividness, positive emotions, and negative emotions, as well as fatigue, vigor, and depressive state, ANCOVAs were carried out with the condition as between-factor and baseline scores as covariates.

Fourth, to analyze the associations between the percentage of time upright and the change in the interpretation bias, mental imagery vividness, positive emotions, negative emotions, fatigue, vigor, and depressive state, Pearson's correlations were performed.

Finally, moderation analyses were carried out to examine whether the relationships between the condition and the post-scores in interpretation bias and mental imagery vividness were moderated by the level of depressive symptomatology. They were performed using the procedure described by Hayes (2018) from the macro PROCESS (version 3.3). In these analyses, the upright condition was coded as " 1 " and the stooped condition as "2". Pre-scores were entered as covariates of the dependent variables in each model. All the regression coefficients were reported in unstandardized form as b-values. Tests of significance $(p<.05)$ or a confidence interval (not including zero) in the interaction "condition $\mathrm{x}$ depressive symptomatology" answered whether the depressive symptomatology moderated the effect of condition on post-scores of interpretation bias or mental imagery vividness. The conditional effects of condition on the post-scores of interpretation bias and mental imagery vividness at medium (the 
mean), low (-1 SD), and high (+1 SD) levels of depressive symptomatology were examined with the pick-a-point approach.

\section{Results}

3.1. Differences in sociodemographic variables, depressive symptomatology and variables investigated in this study

There were no significant differences between conditions for age, $t(52)=1.25, p=.216$; depressive symptomatology, $t(52)=0.18, p=.857$; sex, $\chi^{2}(1, N=54)=0.43, p=.513$; marital status, $\chi^{2}(2, N=54)=1.20, p=.681$; education, $\chi^{2}(2, N=54)=1.33, p=.574$; work status, $\chi^{2}(2, N=54)=1.54, p=.489 ;$ or previous depressive episodes, $\chi^{2}(1, N=$ 54) $=0.26, p=.699($ see Table 1$)$.

Moreover, there were no significant differences between conditions for the scores at baseline of the interpretation bias, $t(52)=-0.68, p=.499 ;$ positive mental imagery vividness, $t(40.59)=-1.19, p=.241$; negative mental imagery vividness, $t(52)$ $=0.03, p=.975$; happy, $t(52)=-0.86, p=.392$; sad, $t(52)=0.31, p=.756$; optimistic, $t(52)=-0.53, p=.601 ;$ hopeless, $t(52)=0.88, p=.381$; anxious, $t(52)=-0.82, p=.417$; relaxed, $t(52)=0.75, p=.456$; fatigue, $t(52)=1.51, p=.137$; vigor, $t(52)=0.14, p=$ $.892 ;$ and depressive state, $t(52)=0.87, p=.389$. 


\section{Table 1}

Descriptive statistics for sociodemographic data and depressive symptomatology

\begin{tabular}{|c|c|c|}
\hline & $\begin{array}{l}\text { Upright condition } \\
\qquad(n=26)\end{array}$ & $\begin{array}{l}\text { Stooped condition } \\
\quad(n=28)\end{array}$ \\
\hline Age (years) $M(S D)$ & $22.50(2.93)$ & $21.54(2.73)$ \\
\hline Sex (\% women) & $69.2 \%$ & $60.7 \%$ \\
\hline \multicolumn{3}{|l|}{ Marital status (\%) } \\
\hline Single & $57.7 \%$ & $64.3 \%$ \\
\hline Married & $3.8 \%$ & $0 \%$ \\
\hline In a relationship & $38.5 \%$ & $35.7 \%$ \\
\hline Education (\%) & $100 \%$ & $100 \%$ \\
\hline Undergraduate students & $80.8 \%$ & $85.7 \%$ \\
\hline Postgraduate students & $7.7 \%$ & $10.7 \%$ \\
\hline Ph.D. students & $11.5 \%$ & $3.6 \%$ \\
\hline \multicolumn{3}{|l|}{ Work status (\%) } \\
\hline Student and employed & $23.1 \%$ & $14.3 \%$ \\
\hline Student & $76.9 \%$ & $82.1 \%$ \\
\hline Unemployed & $0 \%$ & $3.6 \%$ \\
\hline $\begin{array}{l}\text { Previous depressive } \\
\text { episodes (\% yes) }\end{array}$ & $15.4 \%$ & $10.7 \%$ \\
\hline BDI-II $M(S D)$ & $9.19(7.81)$ & $9.57(7.54)$ \\
\hline
\end{tabular}

Note. BDI-II $=$ Beck Depression Inventory-II. 


\subsection{Differences in the posture (thoracic spine flexion)}

An independent-sample t-test showed that there were significant differences in the percentage of time that participants were upright in each condition, $t(47)=8.81, p<$ .001 , Cohen's $d=2.52$. Participants in the upright condition were $85.74 \%$ of the time in an upright posture, while participants in the stooped condition were $14.15 \%$ of the time in an upright posture ${ }^{4}$.

Moreover, considering the average score of the degree inclination of the back, an independent-sample t-test also showed that there were significant differences in this variable, $t(32.27)=-8.30, p<.001$, Cohen's $d=2.39$. The degree of the inclination of the back was significantly more "upright" in the upright condition $(M=3.67 ; S D=$ 3.80) than in the stooped condition $(M=19.40 ; S D=8.43)$.

\subsection{Effects of posture on interpretation bias, mental imagery vividness, and depression-related emotions}

A main effect of condition was found for the interpretation bias, positive emotions (happy and optimistic), and vigor. Participants in the upright condition rated the ambiguous information as more pleasant, experienced more positive emotions, and felt more vigor than participants in the stooped condition. However, there were no main effects of condition for positive or negative mental imagery vividness, negative emotions (hopeless, anxious, sad), relaxation, fatigue, and depressive state (see Table 2 and Figure 2).

\section{Regarding positive emotions, it should be highlighted that the positive ES} (optimistic and happy) and relaxation were computed separately due to the low interitem correlation between optimistic and relaxation $(r<.20)$, and the possibility that

\footnotetext{
${ }^{4}$ Inertial data of 5 participants, 2 in the upright condition and 3 in the stooped condition, were missed, and consequently they were not included in the analyses.
} 
these were therefore not representative of the same content domain. In fact, the ANCOVA results were non-significant when optimistic, happy and relaxation were grouped into the same factor (see the footnote of Table 2). 


\section{Table 2}

ANCOVAs results and values for interpretation bias, mental imagery vividness, emotions, fatigue, vigor, and depressive state.

\begin{tabular}{|c|c|c|c|c|c|c|c|}
\hline & \multicolumn{2}{|c|}{$\begin{array}{c}\text { Upright condition } \\
(n=26)\end{array}$} & \multicolumn{2}{|c|}{$\begin{array}{c}\text { Stooped condition } \\
(n=28)\end{array}$} & \multicolumn{3}{|c|}{ ANCOVAs ${ }^{a}$} \\
\hline & $\begin{array}{c}\text { Pre } \\
M(S D)\end{array}$ & $\begin{array}{c}\text { Post } \\
M(S D)\end{array}$ & $\begin{array}{c}\text { Pre } \\
M(S D)\end{array}$ & $\begin{array}{c}\text { Post } \\
M(S D)\end{array}$ & $\boldsymbol{F}$ & $p$ & $\eta_{p}^{2}$ \\
\hline AST-D-II & $1.33(1.13)$ & $1.42(1.14)$ & $1.54(1.14)$ & $1.31(1.27)$ & $F(1,51)=4.82$ & .033 & .09 \\
\hline \multicolumn{8}{|l|}{ PIT } \\
\hline Positive & $34.35(9.29)$ & $34.96(8.71)$ & $36.86(5.63)$ & $35.54(7.48)$ & $F(1,51)=3.87$ & .055 & .07 \\
\hline Negative & $29.88(8.14)$ & $27.31(8.67)$ & $29.82(6.34)$ & $27.46(7.36)$ & $F(1,51)=0.04$ & .840 & .00 \\
\hline \multicolumn{8}{|l|}{ ES } \\
\hline $\begin{array}{l}\text { Positive (Happy, } \\
\text { Optimistic) }{ }^{\mathrm{b}}\end{array}$ & $3.19(0.87)$ & $3.17(0.95)$ & $3.38(0.91)$ & $2.98(1.23)$ & $F(1,51)=7.69$ & .008 & .13 \\
\hline Relaxed & $3.54(1.03)$ & $3.35(0.98)$ & $3.32(1.09)$ & $3.68(1.16)$ & $F(1,51)=2.50$ & .120 & .05 \\
\hline $\begin{array}{l}\text { Negative (Hopeless, } \\
\text { Anxious, Sad) }\end{array}$ & $1.59(0.73)$ & $1.60(0.60)$ & $1.57(0.59)$ & $1.43(0.61)$ & $F(1,51)=1.97$ & .167 & .04 \\
\hline \multicolumn{8}{|l|}{ POMS } \\
\hline Fatigue & $4.73(4.21)$ & $5.42(4.09)$ & $3.07(3.87)$ & $3.04(3.97)$ & $F(1,51)=2.61$ & .112 & .05 \\
\hline Depression & $3.08(3.60)$ & $2.62(3.09)$ & $2.25(3.40)$ & $2.11(3.52)$ & $F(1,51)=0.14$ & .715 & .00 \\
\hline Vigour & $9.08(4.66)$ & $8.92(4.79)$ & $8.89(5.18)$ & $7.54(5.05)$ & $F(1,51)=6.07$ & .017 & .11 \\
\hline
\end{tabular}

Note. ${ }^{a}$ All ANCOVAs were adjusted for baseline scores. ${ }^{\mathrm{b}}$ When happy, optimistic, relaxed were grouped into the same factor, there was no effect of condition, $F(1,51)=0.25, p=.621, \eta_{p}{ }^{2}=.01$. Upright (Pre: $M=3.31, S D=0.73$; Post: $M=3.23, S D=0.83$ ) and Stooped (Pre: $M=$ 3.36, $S D=0.75$; Post: $M=3.21, S D=1.02)$. AST-D-II = Ambiguous Scenarios Test for depression-related interpretation bias; PIT = Prospective Imagery Task; ES = Emotions' Scale; POMS = The Profile of Mood States. 

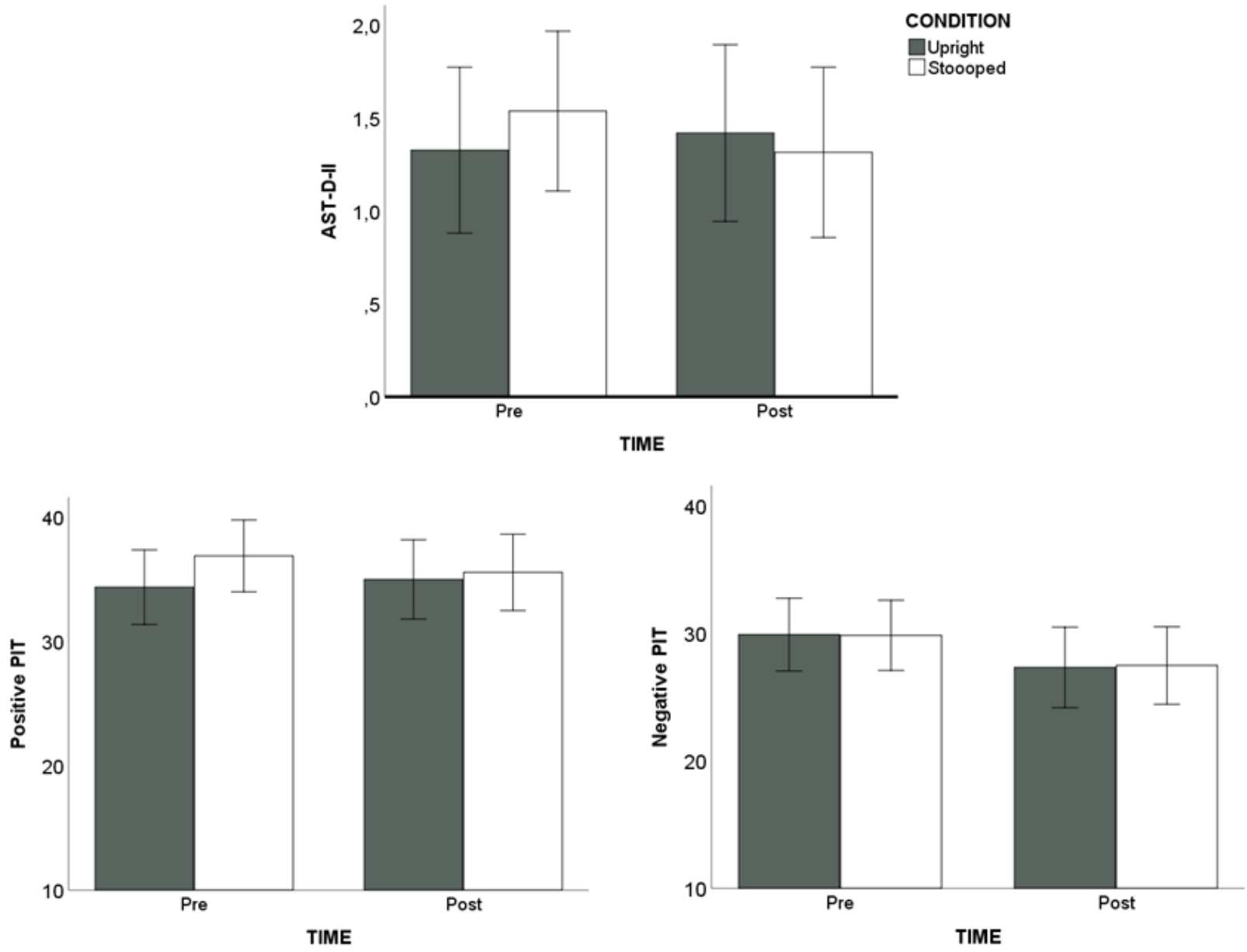

Fig. 2. Graphs of the effect of posture on interpretation bias and mental imagery vividness scores

Note. Error bars represent the $95 \%$ confidence interval of the mean. AST-D-II $=$ Ambiguous Scenarios Test for depression-related interpretation bias; PIT = Prospective Imagery Task. 
3.4. Exploring the interactions between posture, interpretation bias, imagery, and depression-related emotions

3.4.1. Does the percentage of time in an upright posture related to the change in interpretation bias, mental imagery vividness, and depression-related emotions?

Pearson's correlation showed a positive significant relationship between the percentage of time in an upright posture and change in interpretation bias $(r=.35, p=.014)$ and the change in positive mental imagery vividness $(r=.44, p=.002)$. However, the percentage of time in an upright posture was not correlated with change for the other variables: negative mental imagery vividness $(r=-.03, p=.826)$; negative emotions (hopeless, anxious, sad) $(r=.17, p=.235)$; positive emotions (happy and optimistic) $(r$ $=.24, p=.101)$, relaxation $(r=-.18, p=.228)$, fatigue $(r=.18, p=.205)$, depressive state $(r=-.06, p=.679)$, or vigor $(r=.16, p=.266)$.

\subsubsection{Does the depressive symptomatology moderate the effect of body posture on} interpretation bias and mental imagery vividness?

Moderation analyses showed that the depressive symptomatology moderated the effect of condition on the interpretation bias post-scores, but not the positive mental imagery vividness post-scores, $F(1,49)=0.08, p=.785$; or negative mental imagery vividness post-scores, $F(1,49)=0.08, p=.783$. With regards to the interpretation bias model (see Figure 3), the overall model explained $83.85 \%$ of the variance in the interpretation bias post-scores, $F(4,49)=79.77, p<.001$. The interaction between condition and depressive symptomatology was significant, $F(1,49)=4.75, p=.034$, meaning that depressive symptomatology was a moderator of the effect of the condition on the interpretation bias post-scores, accounting for $1.24 \%$ of the variance. Analysis of simple slopes showed that there was a negative significant relationship between condition and the interpretation bias post-scores when the depressive symptomatology was "medium", 
$b=-0.30,95 \%$ CI $[-0.59,-0.01], t=-2.07, p=.044$, and "high", $b=-0.57,95 \%$ CI [$0.91,-0.22], t=-3.27, p=.002$. Participants in the upright (vs. stooped) condition with higher depressive symptomatology achieved higher scores in the interpretation bias post-scores.

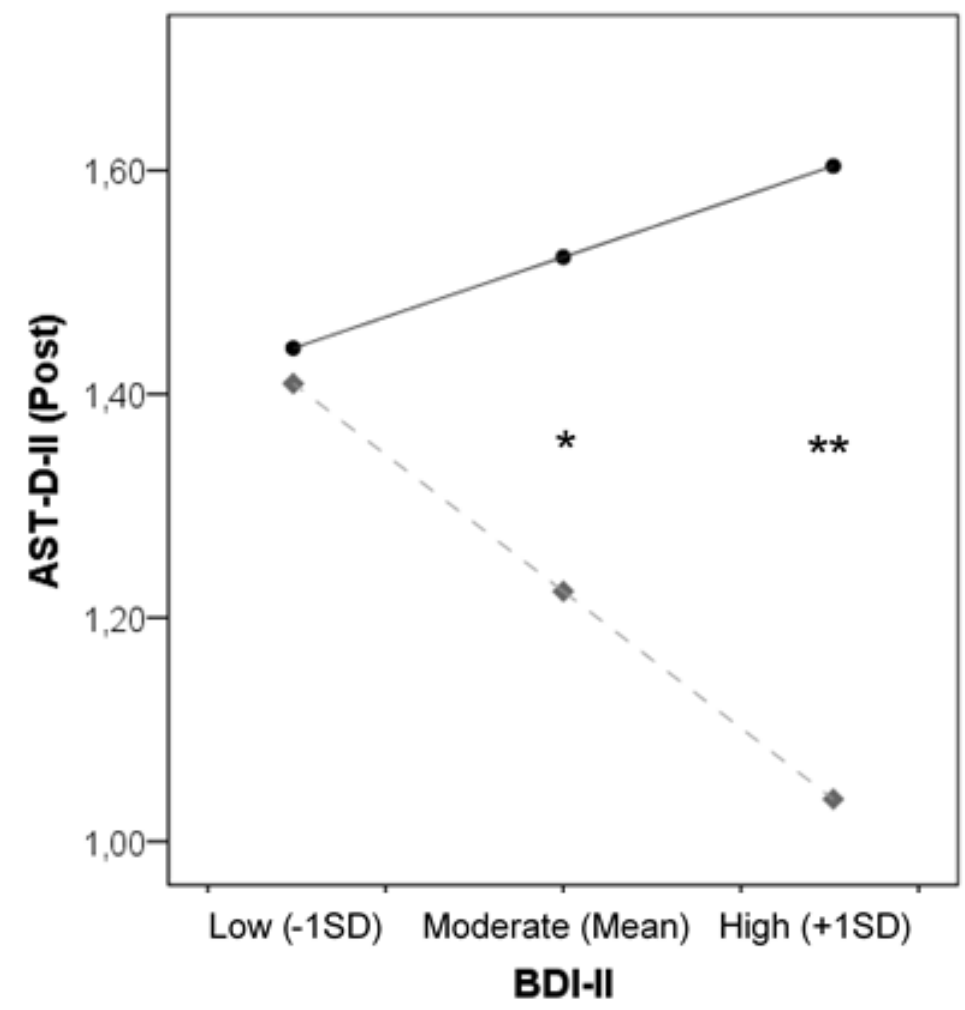

CONDITION

Upright

Stooped

Upright

- - Stooped

Fig. 3. Simple slopes graph of the regression of condition on the interpretation bias post-scores at three levels of the depressive symptomatology (low, medium, high)

Note. BDI-II = Beck Depression Inventory-II. AST-D-II = Ambiguous Scenarios Test for depression-related interpretation bias. "Low", "medium" and "high" levels of the moderators represent the mean and \pm 1 standard deviation (SD) (BDI: $9.39 \pm 7.60)$. Significant $\mathrm{p}$-values $(* p<.05, * * p<.01)$ represent the level of the moderator in which the conditional effect of condition on interpretation bias post-scores is significant. Baseline score is entered as covariate. 


\section{Discussion}

The main objective of this study was to analyze the effect of adopting an upright (vs. stooped) posture on the processing of ambiguous information and the ability to generate mental imagery, as well as on different depression-related emotions. Secondary objectives were to explore the interplay between upright vs. stooped posture, interpretation bias, imagery ability, and emotions.

First, it was verified that there were significant differences between both conditions in the degree of inclination of the thoracic part of the back measured objectively with inertial technology, indicating that the posture of the body was manipulated successfully. Following this manipulation check, partial evidence was found for the main hypothesis of this study. Participants in the upright (vs. stooped) condition interpreted the ambiguous scenarios more positively after adopting their allocated posture. This result is in line with the results found by Michalak et al. (2014), Wilson and Peper (2004) or Tsai et al. (2016), which highlighted the effect of posture in cognitive bias processes. Significant differences were not found between conditions in terms of the ability to imagine negative or positive scenarios, although visual inspection of the graph, and our finding of a correlation between positive imagery vividness and time spent in the upright posture, indicates that the lack of a statistically significant group difference could reflect insufficient power for this particular measure.

Regarding the postural effects on depression-related emotions, participants in the upright (vs. stooped) condition experienced more positive emotions (happiness and optimism) and felt more vigor after the posture manipulation. However, significant between-group differences were not found for relaxation, negative emotions, fatigue or depressive state. This result suggests that the upright posture (vs. stooped) has more effects on positive emotions than on negative emotions, as was found in other studies, 
such as Wilkes et al. (2017). It is also important to highlight the non-significant differences in "relaxation" between conditions, as it indicates that the differences in the interpretation bias are unlikely to be due to the possible "pleasantness" of one posture over the other. According to the direct mechanism of the body on mental states proposed by Körner et al. (2015), it is possible that the upright posture directly influences "positive" ways of processing information or feeling, but not "negative" ways of processing or feeling. This finding may have important clinical implications in the treatment of depressed people, as if these findings were replicated within a clinical sample, the implication would be that postural changes may help to change two symptoms that are relevant in the diagnosis of major depression (American Psychiatric Association, 2013): the lack of positive emotions and/or vigor. Nevertheless, it is important to be cautious with the findings involving the emotion scale (ES), as it has not been previously validated and the effect of condition on positive emotions was only found when excluding the "relaxed" item from the scale on the basis of its low correlations with the other items. It is possible that in the context of this study the rating for "relaxed" was contaminated by the impact on the body of the required posture, as the upright posture required more physical tension in the body.

As regards the secondary exploratory objectives, it was found that the greater the percentage of time participants were in the upright position, the greater the change in interpretation bias and the increase in the vividness of imagined positive scenarios. According to the ICS approach (Barnard and Teasdale, 1991), we suggest that the more time the "body state subsystem" is receiving positive inputs from the posture (e.g., upright posture), the more the positive effects on the "implicational subsystem" (e.g., more positive global view of themselves), leading to interpreting the ambiguous information more positively or imagining the positive scenarios more vividly. However, 
the percentage of time in an upright position does not seem to be associated with changes in positive or negative emotions. Given that the effect of condition on positive emotions was statistically significant, we can speculate that it is not necessary to maintain an upright posture for a long period of time to change positive emotions to a greater extent. However, further studies are required to test this explanation.

In addition, it was found that participants in the upright (vs. stooped) condition with higher levels of depressive symptomatology (at least "mild" scores in the depressive symptomatology) interpreted the ambiguous scenarios more positively after adopting the posture. Thus, this result implies that the effect of posture on interpretation bias is especially relevant for people with at least some minimal level of depressive symptoms (albeit with the caveat that given our sample we cannot generalize to people with severe levels of depression symptoms). Following the ICS approach (Barnard and Teasdale, 1991), it is suggested that when individuals with higher levels of depressive symptomatology hold an upright position, they receive "positive" proprioceptive feedback from the body, and the negative feedback loop between subsystems is disrupted. Consequently, individuals start to process ambiguous information more positively. The ICS approach has also been applied to understand the therapeutic effects of mindfulness on depression (Teasdale et al., 1995). However, the level of depressive symptomatology did not moderate the effect of posture on the vividness of positive imagery, suggesting the levels of depressive symptomatology does not have a relevant role in the effect of posture on the generation of positive imagery.

To summarize the overall pattern of results, a consistent association between posture and interpretation bias was found, indicated by both the between-group differences, correlational, and moderation analysis. A consistent lack of association across analyses and outcome measures was found between posture and "negative" 
outcomes, such as negative imagery vividness and negative emotional states. A more mixed pattern was found for positive imagery vividness (correlation only) and positive emotional states (group difference only). This may reflect differences in the strength or nature (e.g. not so strongly dose-response for emotions) of the relationships between these outcomes and posture (as noted above), but in the absence of replication these more mixed findings should be interpreted more cautiously. Moreover, the results should be interpreted with caution because only a small proportion of a larger number of analyses were statistically significant, and the results could be inflated by chance.

This study has theoretical implications, as it supports the theories of embodied emotion and cognition, that is, the role of the body in emotional and cognitive processes (Winkielman et al., 2015). In this regard, this study also highlights the importance of including the body's role in the cognitive models of depression, as the findings point out that posture interacts with the mechanisms that Holmes et al. (2009) suggest are involved in the maintenance of depression.

Moreover, these findings may have clinical implications, as results suggest that manipulating posture may modify mechanisms that are involved in the onset and maintenance of depression. In this sense, it could be relevant to include the manipulation of the body during the interventions such as those using cognitive bias modification $(\mathrm{CBM})$ procedures. $\mathrm{CBM}$ interventions are aimed at modifying information-processing biases through a computerized repeated practice that reinforces a more positive style of information processing, in which participants repeatedly practice imagining positive resolutions for ambiguous situations (e.g., Blackwell and Holmes, 2010; Blackwell et al., 2015). Hence, future studies should explore the effects of manipulating body posture while completing CBM. 
Limitations of this study should be noted. First, the study was carried out with a non-clinical sample, restricting the generalizability to clinical depressed samples. Nevertheless, the results of the moderation analysis point out that the effect of posture is stronger at higher symptoms of depression. Consequently, we consider that this encouraging finding justifies exploring in future studies whether an upright posture leads to processing ambiguous information in a more positive way in a clinical sample of individuals with major depression. Second, the effects of posture on these mechanisms were not verified in the mid- or in the long-term. Longitudinal studies would help to clarify how long this effect lasts. Third, this study did not have a condition of individuals adopting their usual posture. Such a condition would help to distinguish whether the upright posture has benefits over the usual posture that individuals adopt.

\section{Conclusions}

The body posture interacts with mechanisms involved in the maintenance of depression (interpretation bias and vividness of positive imagery), as well as with depressionrelated emotions (happiness, optimism, and vigor). This study has theoretical and clinical implications that should be continued explored to clarify the role of the posture in depression.

\section{References}

American Psychiatric Association, 2013. Diagnostic and statistical manual of mental disorders (DSM-5®). American Psychiatric Association, Arlington.

Andrade, E., Arce, C., De Francisco, C., Torrado, J., Garrido, J., 2013. Versión breve en español del cuestionario POMS para deportistas adultos y población general 
[Abbreviated version in Spanish of the POMS questionnaire for adult athletes and general population]. Rev Psicol Deporte, 22(1), 59-102.

Barnard, P. J., Teasdale, J. D., 1991. Interacting cognitive subsystems: A systemic approach to cognitive-affective interaction and change. Cogn Emot, 5(1), 1-39. doi: $10.1080 / 02699939108411021$

Barsalou, L. W, 2008. Grounded cognition. Annu Rev Psychol, 59, 617-645. doi: 10.1146/annurev.psych.59.103006.093639

Blackwell, S. E., Holmes, E. A., 2010. Modifying interpretation and imagination in clinical depression: A single case series using cognitive bias modification. Appl Cogn Psychol, 24(3), 338-350. doi: 10.1002/acp.1680

Blackwell, S. E., Browning, M., Mathews, A., Pictet, A., Welch, J., Davies, J., Watson, P., Geddes, J.R., Holmes, E. A., 2015. Positive imagery-based cognitive bias modification as a web-based treatment tool for depressed adults: a randomized controlled trial. Clin Psychol Sci, 3(1), 91-111. doi: 10.1177/2167702614560746

Blackwell, S. E., Westermann, K., Woud, M. L., Cwik, J. C., Neher, T., Graz, C., Nyhuis, P.W. Margraf, J., 2018. Computerized positive mental imagery training versus cognitive control training versus treatment as usual in inpatient mental health settings: study protocol for a randomized controlled feasibility trial. Pilot Feasibility Stud, 4(1), 133.

Beck, A. T., Steer, R. A., Brown, G. K., 1996. Beck Depression Inventory Manual. The Psychological Corporation, San Antonio.

Carney, D. R., Cuddy, A. J., Yap, A. J., 2010. Power posing: Brief nonverbal displays affect neuroendocrine levels and risk tolerance. Psychol Sci, 21(10), 1363-1368. doi: $10.1177 / 0956797610383437$ 
Clark, D. A., Beck, A. T., Alford B.A., 1999. Scientific foundations of cognitive theory and therapy of depression. Wiley, New York.

Cuddy, A. J. C., Schultz, S. J., Fosse, N. E., 2018. P-Curving a More Comprehensive Body of Research on Postural Feedback Reveals Clear Evidential Value For Power-Posing Effects: Reply to Simmons and Simonsohn (2017). Psychol Sci, 29(4), 656-666. doi: 10.1177/0956797617746749

Cuddy, A. J., Wilmuth, C. A., Yap, A. J., Carney, D. R., 2015. Preparatory power posing affects nonverbal presence and job interview performance. J Appl Psychol, 100(4), 1286. doi: 10.1037/a0038543

Everaert, J., Koster, E. H., Derakshan, N., 2012. The combined cognitive bias hypothesis in depression. Clin Psychol Rev, 32(5), 413-424. doi: 10.1016/j.cpr.2012.04.003

Everaert, J., Podina, I. R., Koster, E. H., 2017. A comprehensive meta-analysis of interpretation biases in depression. Clin Psychol Rev, 58, 33-48. doi: 10.1016/j.cpr.2017.09.005

Faul, F., Erdfelder, E., Lang, A. G., Buchner, A., 2007. G* Power 3: A flexible statistical power analysis program for the social, behavioral, and biomedical sciences. Behav Res Methods, 39(2), 175-191.

Gotlib, I. H., Joormann, J., 2010. Cognition and depression: current status and future directions. Annu Rev Clin Psychol, 6, 285-312. doi: 10.1146/annurev.clinpsy.121208.131305

Hayes, A. F. (2018). Introduction to mediation, moderation, and conditional process analysis: A regression-based approach (2n edition). New York: Guilford Press.

Holmes, E. A., Blackwell, S. E., Burnett-Heyes, S., Renner, F., Raes, F., 2016. Mental imagery in depression: phenomenology, potential mechanisms, and treatment 
implications. Annu Rev Clin Psychol, 12, 249-280. doi: 10.1146/annurev-clinpsy021815-092925

Holmes, E. A., Lang, T. J., Deeprose, C., 2009. Mental imagery and emotion in treatment across disorders: Using the example of depression. Cogn Behav Ther, 38(S1), 21-28. doi: 10.1080/16506070902980729

Holmes, E. A., Lang, T. J., Moulds, M. L., Steele, A. M., 2008. Prospective and positive mental imagery deficits in dysphoria. Behav Res Ther, 46(8), 976-981. doi: 10.1016/j.brat.2008.04.009

Ingram, R. E., 1984. Toward an information-processing analysis of depression. Cogn Ther Res, 8(5), 443-477. doi: 10.1007/BF01173284

Joormann, J., Yoon, K. L., Zetsche, U., 2007. Cognitive inhibition in depression. Appl Prev Psychol, 12(3), 128-139. doi: 10.1016/j.appsy.2007.09.002

Körner, A., Topolinski, S., Strack, F., 2015. Routes to embodiment. Front Psychol, 6, 940. doi: 10.3389/fpsyg.2015.00940

LeMoult, J., Gotlib, I. H., 2019. Depression: a cognitive perspective. Clin Psychol Rev, 69, 51-66. doi: 10.1016/j.cpr.2018.06.008

Piedmont, R. L., 2014. Inter-item correlations, in: Michalos, A.C. (Ed.), Encyclopedia of quality of life and well-being research. Springer, Dordrecht, The Netherlands, pp. 3303-3304.

Platt, B., Waters, A. M., Schulte-Koerne, G., Engelmann, L., Salemink, E., 2017. A review of cognitive biases in youth depression: attention, interpretation and memory. Cogn Emot, 31(3), 462-483. doi: 10.1080/02699931.2015.1127215

Mathews, A., MacLeod, C., 2005. Cognitive vulnerability to emotional disorders. Annu Rev Clin Psychol, 1, 167-195. doi: 10.1146/annurev.clinpsy.1.102803.143916 
McNair, D. M., Lorr, M., Droppleman, L. F., 1971. Profile of mood state manual. Educational and Industrial Testing Service, San Diego.

Michalak, J., Mischnat, J., Teismann, T., 2014. Sitting posture makes a differenceembodiment effects on depressive memory bias. Clin Psychol Psychother, 21(6), 519-524. doi: 10.1002/cpp.1890

Nair, S., Sagar, M., Sollers III, J., Consedine, N., Broadbent, E., 2015. Do slumped and upright postures affect stress responses? A randomized trial. Health Psychol, 34(6), 632. doi: 10.1037/hea0000146

Niedenthal, P. M., 2007. Embodying emotion. Science, 316(5827), 1002-1005. doi: $10.1126 /$ science. 1136930

Rohrbacher, H., Reinecke, A., 2014. Measuring change in depression-related interpretation bias: development and validation of a parallel ambiguous scenarios test. Cogn Behav Ther, 43(3), 239-250. doi: 10.1080/16506073.2014.919605

Sanz, J., Perdigón, A. L., Vázquez, C., 2003. Adaptación española del Inventario para la Depresión de Beck-II (BDI-II): 2. Propiedades psicométricas en población general [Spanish adaptation of the Beck-II Depression Inventory (BDI-II): 2.

Psychometric properties in the general population]. Clínica y Salud, 14(3), 249280.

Stöber, J., 2000. Prospective cognitions in anxiety and depression: Replication and methodological extension. Cogn Emot, 14(5), 725-729. doi: $10.1080 / 02699930050117693$

Teasdale, J. D., Segal, Z., Williams, J. M. G., 1995. How does cognitive therapy prevent depressive relapse and why should attentional control (mindfulness) training help?. Behav Res Ther, 33(1), 25-39. doi: 10.1016/0005-7967(94)E0011-7 
Tsai, H. Y., Peper, E., Lin, I. M., 2016. EEG patterns under positive/negative body postures and emotion recall tasks. NeuroRegulation, 3(1), 23. doi: $10.15540 / \mathrm{nr} .3 .1 .23$

Veenstra, L., Schneider, I. K., Koole, S. L., 2016. Embodied mood regulation: the impact of body posture on mood recovery, negative thoughts, and moodcongruent recall. Cogn Emot, 1-16. doi: 10.1080/02699931.2016.1225003

Watson, D., Clark, L. A., Tellegen, A., 1988. Development and validation of brief measures of positive and negative affect: the PANAS scales. J Pers Soc Psychol, 54(6), 1063. doi: 10.1037/0022-3514.54.6.1063

Wilkes, C., Kydd, R., Sagar, M., Broadbent, E., 2017. Upright posture improves affect and fatigue in people with depressive symptoms. J Behav Ther Exp Psychiatry, 54, 143-149. doi: 10.1016/j.jbtep.2016.07.015

Williams, J. M. G., Watts, F. N., MacLeod, C., Mathews, A., 1988. Cognitive psychology and emotional disorders. John Wiley \& Sons, New York.

Williams, J. M. G., Watts, F. N., MacLeod, C., Mathews, A., 1997. Cognitive psychology and emotional disorders. John Wiley \& Sons, Chichester.

Wilson, V. E., Peper, E., 2004. The effects of upright and slumped postures on the recall of positive and negative thoughts. Appl Psychophysiol Biofeedback, 29(3), 189195. doi: 10.1023/B:APBI.0000039057.32963.34

Wilkes, C., Kydd, R., Sagar, M., Broadbent, E., 2017. Upright posture improves affect and fatigue in people with depressive symptoms. J Behav Ther Exp Psychiatry, 54, 143-149. doi: 10.1016/j.jbtep.2016.07.015

Winkielman, P., Niedenthal, P. M., Wielgosz, J., Eelen, J., Kavanagh, L. C., 2015. Embodiment of cognition and emotion, in: Mikulincer, M., Shaver, P. R., Borgida, E, Bargh, J. A. (Eds.), APA handbooks of psychology, APA handbook 
of personality and social psychology, Vol. 1: Attitudes and social cognition. American Psychological Association, Washington DC, pp. 151-175.

World Health Organization, 2017. Depression and Other Common Mental Disorders:

Global Health Estimates. World Health Organization, Geneva 


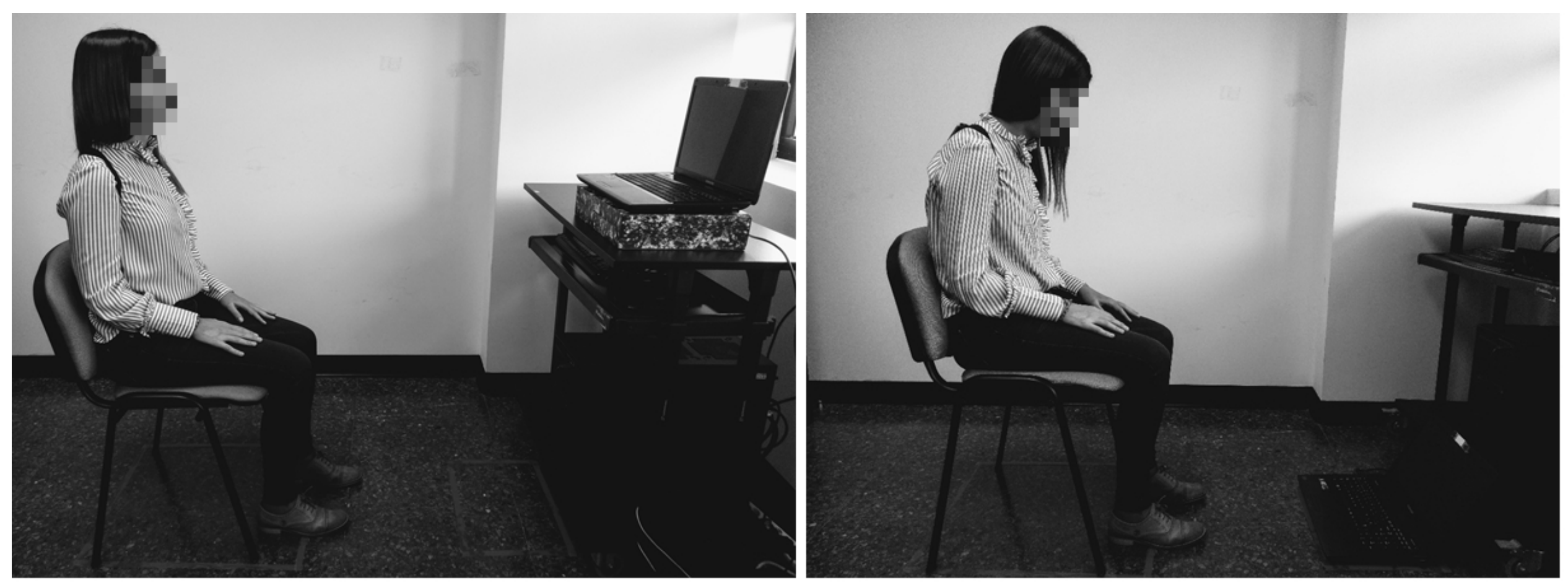



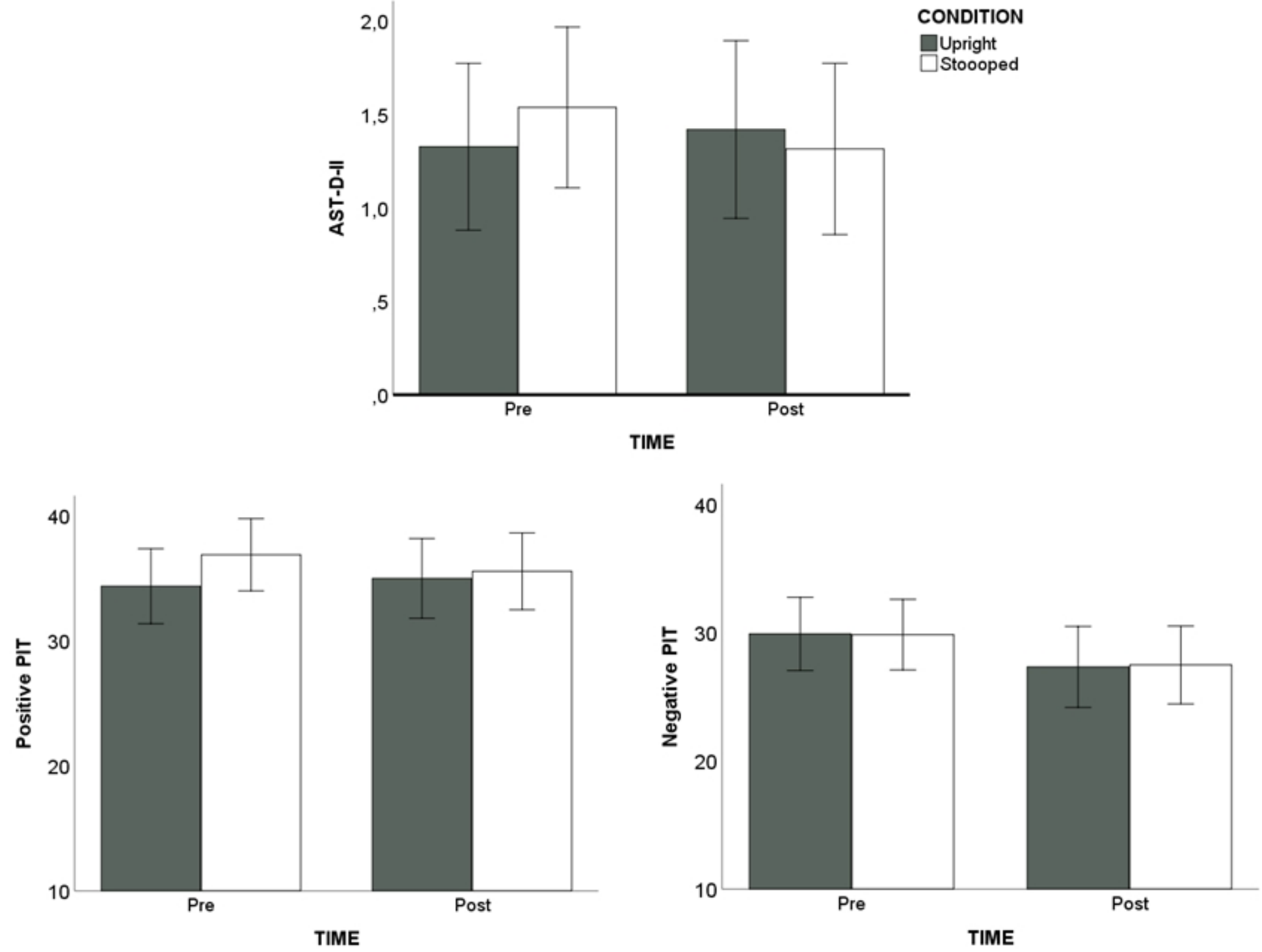


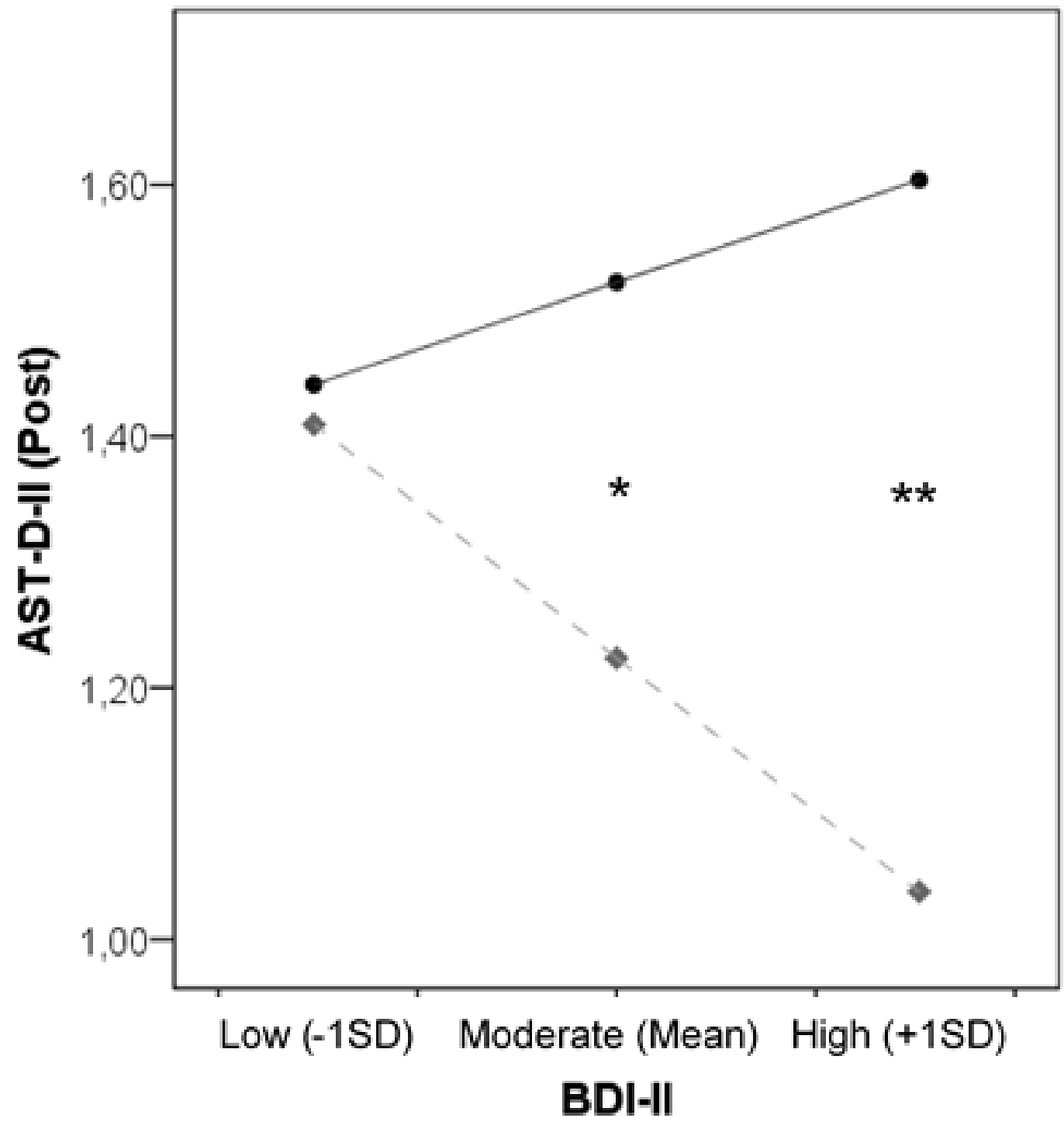

\section{CONDITION}

Upright

Stooped

- Upright

- -Stooped 
Table 1

Descriptive statistics for sociodemographic data and depressive symptomatology

\begin{tabular}{lcc}
\hline & $\begin{array}{c}\text { Upright condition } \\
(n=26)\end{array}$ & $\begin{array}{c}\text { Stooped condition } \\
(n=28)\end{array}$ \\
\hline Age (years) $\boldsymbol{M}(\boldsymbol{S D})$ & $22.50(2.93)$ & $21.54(2.73)$ \\
Sex (\% women) & $69.2 \%$ & $60.7 \%$ \\
Marital status (\%) & & \\
Single & $57.7 \%$ & $64.3 \%$ \\
Married & $3.8 \%$ & $0 \%$ \\
In a relationship & $38.5 \%$ & $35.7 \%$ \\
Education (\%) & $100 \%$ & $100 \%$ \\
Undergraduate students & $80.8 \%$ & $85.7 \%$ \\
Postgraduate students & $7.7 \%$ & $10.7 \%$ \\
Ph.D. students & $11.5 \%$ & $3.6 \%$ \\
Work status (\%) & & $10.7 \%$ \\
Student and employed & $23.1 \%$ & $9.57(7.54)$ \\
Student & $76.9 \%$ & $3.6 \%$ \\
Unemployed & $0 \%$ & $14.3 \%$ \\
Previous depressive \\
episodes (\% yes)
\end{tabular}

Note. BDI-II $=$ Beck Depression Inventory-II. 
Table 2

ANCOVAs results and values for interpretation bias, mental imagery vividness, emotions, fatigue, vigor, and depressive state.

\begin{tabular}{|c|c|c|c|c|c|c|c|}
\hline & \multicolumn{2}{|c|}{$\begin{array}{c}\text { Upright condition } \\
(n=26)\end{array}$} & \multicolumn{2}{|c|}{$\begin{array}{c}\text { Stooped condition } \\
(n=28)\end{array}$} & \multicolumn{3}{|c|}{$\operatorname{ANCOVAs}^{\mathrm{a}}$} \\
\hline & $\begin{array}{c}\text { Pre } \\
M(S D)\end{array}$ & $\begin{array}{c}\text { Post } \\
M(S D)\end{array}$ & $\begin{array}{c}\text { Pre } \\
M(S D)\end{array}$ & $\begin{array}{c}\text { Post } \\
M(S D)\end{array}$ & $F$ & $p$ & $\boldsymbol{\eta}_{p}^{2}$ \\
\hline AST-D-II & $1.33(1.13)$ & $1.42(1.14)$ & $1.54(1.14)$ & $1.31(1.27)$ & $F(1,51)=4.82$ & .033 & .09 \\
\hline \multicolumn{8}{|l|}{ PIT } \\
\hline Positive & 34.35 (9.29) & $34.96(8.71)$ & $36.86(5.63)$ & $35.54(7.48)$ & $F(1,51)=3.87$ & .055 & .07 \\
\hline Negative & $29.88(8.14)$ & $27.31(8.67)$ & $29.82(6.34)$ & $27.46(7.36)$ & $F(1,51)=0.04$ & .840 & .00 \\
\hline \multicolumn{8}{|l|}{ ES } \\
\hline $\begin{array}{l}\text { Positive (Happy, } \\
\text { Optimistic) }\end{array}$ & $3.19(0.87)$ & $3.17(0.95)$ & $3.38(0.91)$ & $2.98(1.23)$ & $F(1,51)=7.69$ & .008 & .13 \\
\hline Relaxed & $3.54(1.03)$ & $3.35(0.98)$ & $3.32(1.09)$ & $3.68(1.16)$ & $F(1,51)=2.50$ & .120 & .05 \\
\hline $\begin{array}{l}\text { Negative (Hopeless, } \\
\text { Anxious, Sad) }\end{array}$ & $1.59(0.73)$ & $1.60(0.60)$ & $1.57(0.59)$ & $1.43(0.61)$ & $F(1,51)=1.97$ & .167 & .04 \\
\hline \multicolumn{8}{|l|}{ POMS } \\
\hline Fatigue & $4.73(4.21)$ & $5.42(4.09)$ & $3.07(3.87)$ & $3.04(3.97)$ & $F(1,51)=2.61$ & .112 & .05 \\
\hline Depression & $3.08(3.60)$ & $2.62(3.09)$ & $2.25(3.40)$ & $2.11(3.52)$ & $F(1,51)=0.14$ & .715 & .00 \\
\hline Vigour & $9.08(4.66)$ & $8.92(4.79)$ & $8.89(5.18)$ & $7.54(5.05)$ & $F(1,51)=6.07$ & .017 & .11 \\
\hline
\end{tabular}

Note. ${ }^{a}$ All ANCOVAs were adjusted for baseline scores. ${ }^{b}$ When happy, optimistic, relaxed were grouped into the same factor, there was no effect of condition, $F(1,51)=0.25, p=.621, \eta_{p}{ }^{2}=.01$. Upright (Pre: $M=3.31, S D=0.73$; Post: $M=3.23$, $S D=0.83$ ) and Stooped (Pre: $M=$ 3.36, $S D=0.75$; Post: $M=3.21, S D=1.02)$. AST-D-II = Ambiguous Scenarios Test for depression-related interpretation bias; PIT = Prospective Imagery Task; ES = Emotions' Scale; POMS = The Profile of Mood States. 


\section{Conflict of interest}

All authors declare no conflicts of interest. 


\section{Author Contribution Statement}

MM: Conceptualization, Methodology, Data curation, Formal analysis, Investigation,

Writing - original draft, Writing - review \& editing

AB: Data curation, Formal analysis, Software, Writing - review \& editing

AE: Conceptualization, Methodology, Supervision, Writing - review \& editing

EE: Conceptualization, Methodology, Supervision, Writing - review \& editing

JNS: Conceptualization, Investigation, Writing - review \& editing

RLL: Data curation, Formal analysis, Software, Writing - review \& editing

SB: Conceptualization, Methodology, Supervision, Writing - review \& editing

RB: Conceptualization, Funding acquisition, Methodology, Resources, Supervision,

Writing - review \& editing 